\title{
Mechanical Lamb-shift analogue for the Cooper-pair box
}

\author{
A. D. Armour, ${ }^{a}$ M. P. Blencowe, ${ }^{b, *}$ K. C. Schwab ${ }^{c}$

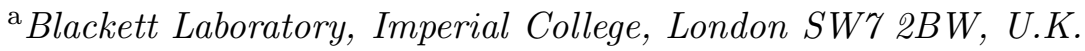 \\ ${ }^{\mathrm{b}}$ Department of Physics and Astronomy, Dartmouth College, Hanover, New \\ Hampshire 03755 \\ ${ }^{\mathrm{c}}$ Laboratory for Physical Sciences, College Park, Maryland 20740
}

\begin{abstract}
We estimate the correction to the Cooper-pair box energy level splitting due to the quantum motion of a coupled micromechanical gate electrode. While the correction due to zero-point motion is very small, it should be possible to observe thermal motion-induced corrections to the photon-assisted tunneling current.
\end{abstract}

Key words: Cooper-pair box, Micromechanical systems

PACS: $73.50 .+\mathrm{r}, 85.85 .+\mathrm{j}$

In a recent experiment [1], the coherent control of macroscopic quantum superposition states in a Cooper box was demonstrated. This represents an important advance towards the realization of a solid state quantum computer [2]. The ability to manipulate the Cooper box states also allows the possibility of producing entangled states between the Cooper box and any other

* Corresponding author: Fax: +(603)-646-1446, email: m.blencowe@dartmouth.edu 
dynamical system, possibly macroscopic, to which it can be coupled. Examples include coupling the Cooper box to another large superconducting island [3], a superconducting resonator[4] and to a micromechanical gate electrode [5], which could take the form of a cantilever or bridge-like structure. In the present work, we examine the effect of a mechanical gate electrode on the energy levels of a Cooper box. In particular, we consider what might be viewed as a mechanical analogue of the Lamb shift, in which the quantum zero-point motion of the mechanical oscillator modifies the level separation between the Cooper box states.

The Hamiltonian for the Cooper box-coupled mechanical gate electrode system is

$$
H=4 E_{\mathrm{C}}\left[n_{\mathrm{g}}-(n+1 / 2)\right] \hat{\sigma}_{z}-\frac{1}{2} E_{\mathrm{J}} \hat{\sigma}_{x}+\hbar \omega \hat{a}^{\dagger} \hat{a}-4 E_{\mathrm{C}} n_{\mathrm{g}}^{\mathrm{m}} \frac{\Delta x_{\mathrm{zp}}}{d}\left(\hat{a}+\hat{a}^{\dagger}\right) \hat{\sigma}_{z},
$$

where $E_{\mathrm{C}}=e^{2} / 2 C_{\mathrm{J}}$ is the single-electron charging energy, $n_{\mathrm{g}}=-\left(C_{\mathrm{g}}^{\mathrm{c}} V_{\mathrm{g}}^{\mathrm{c}}+\right.$ $\left.C_{\mathrm{g}}^{\mathrm{m}} V_{\mathrm{g}}^{\mathrm{m}}\right) / 2 e$ is the dimensionless, total gate charge with control gate voltage $V_{\mathrm{g}}^{\mathrm{c}}$ and mechanical gate electrode voltage $V_{\mathrm{g}}^{\mathrm{m}}$ chosen such that $n_{\mathrm{g}}$ is close to $n+1 / 2$ for some $n$ (so that only Cooper charge states $|n\rangle \equiv\left(\begin{array}{l}1 \\ 0\end{array}\right)$ and $|n+1\rangle \equiv\left(\begin{array}{l}0 \\ 1\end{array}\right)$ play a role), $n_{\mathrm{g}}^{\mathrm{m}}=-C_{\mathrm{g}}^{\mathrm{m}} V_{\mathrm{g}}^{\mathrm{m}} / 2 e, E_{\mathrm{J}}$ is the Josephson coupling energy, $\Delta x_{\mathrm{zp}}$ is the zero-point displacement uncertainty of the mechanical gate electrode, $d$ is the mechanical electrode-island gap, and $\omega$ is the frequency of the fundamental flexural mode of the mechanical electrode. We assume $C_{\mathrm{J}} \gg C_{\mathrm{g}}$ and $d \gg \Delta x_{\mathrm{zp}}$.

Neglecting the coupling between the Cooper box and mechanical electrode, the energy eigenvalues are $E_{0, N}^{(0)}=-\Delta E(\eta) / 2+N \hbar \omega$ and $E_{1, N}^{(0)}=+\Delta E(\eta) / 2+$ $N \hbar \omega$, where $\Delta E(\eta)=E_{\mathrm{J}} / \sin \eta$ with the mixing angle $\eta=\tan ^{-1}\left(E_{\mathrm{J}} /\left[8 E_{\mathrm{C}}(n+\right.\right.$ 
$\left.\left.\left.1 / 2-n_{\mathrm{g}}\right)\right]\right)$. To second order in the coupling, we have

$$
\begin{aligned}
E_{1, N}^{(2)}-E_{0, M}^{(2)} & =\Delta E(\eta)\left[1+32(N+M+1)\left(\frac{\Delta x_{\mathrm{zp}}}{d}\right)^{2}\right. \\
& \left.\times\left(\frac{E_{\mathrm{C}}^{2}}{(\Delta E(\eta))^{2}-(\hbar \omega)^{2}}\right) \sin ^{2} \eta n_{\mathrm{g}}^{\mathrm{m} 2}\right]+(N-M) \hbar \omega .
\end{aligned}
$$

Notice that we require $\eta \neq 0$ in order for the coupling to modify the energy levels. If $E_{\mathrm{J}}=0$, then the only effect of the coupling of the Cooper box to the mechanical oscillator is a shift of the harmonic potential to the left or to the right, depending on the state of the Cooper box. With $E_{\mathrm{J}} \neq 0$ and $\hbar \omega<\Delta E(\eta)$, we see that the interaction with the mechanical oscillator increases the gap between the Cooper box levels.

Let us now estimate the magnitude of the possible gap increase under realisable conditions, supposing the mechanical oscillator to be in its ground state and assuming also that $\hbar \omega \ll \Delta E(\eta)$. Josephson energies for Cooper boxes are typically in the tens of micro-eV range, translating to tens of $\mathrm{GHz}$ which exceeds by at least an order of magnitude the fundamental frequencies of realisable micromechanical oscillators. For the oscillator undergoing zero-point motion $(N=M=0)$, the gap increase is then approximately $32 n_{\mathrm{g}}^{\mathrm{m} 2}\left(\Delta x_{\mathrm{zp}} / d\right)^{2}\left(E_{\mathrm{C}} / E_{\mathrm{J}}\right)^{2} \sin ^{4} \eta$. Considering, for example, $E_{\mathrm{C}}=100 \mu \mathrm{eV}$, $E_{\mathrm{J}}=10 \mu \mathrm{eV}, n+1 / 2-n_{\mathrm{g}}=0.01, \Delta x_{\mathrm{zp}}=10^{-2} \AA, d=0.1 \mu \mathrm{m}$, and $n_{\mathrm{g}}^{\mathrm{m}}=10$, the gap increase is about $10^{-5}$, likely too small to be detected.

If, on the other hand, the mechanical oscillator is in a thermal state, then for the same parameter values the gap increase is approximately $10^{-5}(2 \bar{N}+1)$, where $\bar{N}$ is the thermal-averaged occupation number. Considering, for example, a fundamental frequency $\nu=50 \mathrm{kHz}$ and temperature $T=30 \mathrm{mK}$, we have $\bar{N} \approx 2.5 \times 10^{4}$ giving a significant gap increase of about 0.25 . 
A possible way to probe the effect of the mechanical oscillator thermal motion on the Cooper box levels is measure the photon-assisted Josephson quasiparticle (PAJQP) tunneling current dependence on total gate charge $n_{\mathrm{g}}[6]$. As the mechanical gate voltage $V_{\mathrm{g}}^{\mathrm{m}}$ is turned on, increasing $n_{\mathrm{g}}^{\mathrm{m}}$, we would expect the PAJQP current peak to the left (right) of the main Josephson quasiparticle (JQP) tunneling current peak to shift towards the right (left), signalling the increasing gap between the $n$ and $n+1$ Cooper box levels. At the same time, the PAJQP peaks should broaden due to the thermal motion of the mechanical oscillator.

A proper analysis of the quasiparticle tunneling current is required which includes the corrections to the Cooper box energy levels due to the coupling to the mechanical oscillator. A suitable starting point is the analysis of the experiment of Nakamura et al. [1] given in Ref. [7]. This will be the subject of a future investigation.

This work was supported in part by the NSA and ARDA under ARO contract number DAAG190110696, and by the EPSRC under Grant No. GR/M42909/01.

\section{References}

[1] Y. Nakamura, Yu. A. Pashkin and J. S. Tsai, Nature 398 (1999), 786.

[2] Y. Makhlin, G. Schön and A. Shnirman, Rev. Mod. Phys. 73 (2001) 357.

[3] F. Marquardt and C. Bruder, Phys. Rev. B 63 (2001) 054514.

[4] O. Buisson and F. W. J. Hekking, cond-mat/0008275.

[5] A. D. Armour, M. P. Blencowe and K. C. Schwab (in preparation). 
[6] Y. Nakamura, C. D. Chen and J. S. Tsai, Phys. Rev. Lett. 79 (1997) 2328.

[7] M-S Choi, R. Fazio, J. Siewert and C. Bruder Europhys. Lett. 53 (2001) 251. 